\title{
On arithmetic progressions with equal products
}

\author{
by \\ N. Saradha (Bombay), T. N. Shorey (Bombay) \\ and R. TiJdeman (Leiden) \\ To Wolfgang M. Schmidt \\ at the occasion of his sixtieth birthday
}

1. Introduction. In this paper we consider the diophantine equation

$$
x\left(x+d_{1}\right) \ldots\left(x+(L-1) d_{1}\right)=y\left(y+d_{2}\right) \ldots\left(y+(M-1) d_{2}\right)
$$

in positive integers $d_{1}, d_{2}, L>1, M>1, x, y$. We assume throughout the paper that $d_{1}$ and $d_{2}$ are fixed and that $L / M$ has a given ratio. Put $k=\operatorname{gcd}(L, M), l=L / k$ and $m=M / k$. Hence $l$ and $m$ are fixed and $\operatorname{gcd}(l, m)=1$.

There are several results in the literature in case $d_{1}=d_{2}=1$. In 1963 Mordell [6] proved that (1) with $(L, M)=(2,3)$ implies that $(x, y)=(2,1)$ or $(14,5)$. MacLeod and Barrodale [5] showed in 1970 that (1) has no solutions if $(L, M)=(2,4),(2,6),(2,8),(2,12),(4,8)$ or $(5,10)$ and admits only the solution $(x, y)=(8,1)$ if $(L, M)=(3,6)$. Two years later Boyd and Kisilevsky [1] proved that $(x, y)=(2,1),(4,2),(55,19)$ are the only solutions of (1) if $(L, M)=(3,4)$. For fixed $L$ and $M=2 L$, MacLeod and Barrodale further showed that (1) admits only finitely many solutions. In 1990 Saradha and Shorey [8] proved that there exists only one solution with $M=2 L$, namely $(L, M, x, y)=(3,6,8,1)$. In 1991 they showed in [9] that (1) has no solutions with $M=3 L$ or $M=4 L$. Recently, Mignotte and Shorey showed that this is also the case when $M=5 L$ or $M=6 L$. In general, for $m>1$ and $M=m L$, Saradha and Shorey [10] proved that equation (1) implies that $\max (L, x, y)$ is bounded by an effectively computable number depending only on $m$.

In 1992 Saradha and Shorey [11] started the study of equation (1) for more general pairs $\left(d_{1}, d_{2}\right)$. They showed that if $d_{1}=d_{2}=d$ and $l=1, m>$ 1 then there exists an effectively computable upper bound for $k=L, x$ and $y$ which depends only on $d$ and $m$. Later, Saradha and Shorey [12] showed 
that equation (1) implies that $k$ is bounded by an effectively computable number depending only on $d_{1}, d_{2}$ and $m$. They further showed that $x$ and $y$ are bounded by such a number unless

(i) $m=k=2, d_{1}=2 d_{2}^{2}, x=y^{2}+3 d_{2} y$, or

(ii) $d_{1} / d_{2}^{m}$ is a product of $m>2$ distinct positive integers composed of primes not exceeding $m$ and $m \geq \alpha(k)$ where $\alpha(k)=14$ for $2 \leq k \leq 7$, $\alpha(8)=50$ and $\alpha(k)=\exp (k \log k-1.25475 k-\log k+1.56577)$ for $k \geq 9$.

Condition (i) is necessary. In the present paper we shall show that condition (ii) is superfluous.

The authors [13] studied equation (1) with $L=M$. Observe that in this case $d_{1}=d_{2}$ implies $x=y$. Therefore there is no loss of generality in assuming that $d_{1}<d_{2}$ and $\operatorname{gcd}\left(d_{1}, d_{2}, x, y\right)=1$. We proved that under these assumptions $L, M, x$ and $y$ are bounded by an effectively computable number depending only on $d_{2}$ unless $d_{1}=1, d_{2}=4, x=L+1, y=2$. Observe that

$$
(L+1)(L+2) \ldots(2 L)=2 \cdot 6 \cdot \ldots \cdot(4 L-2) \quad \text { for } L=2,3, \ldots,
$$

since both sides equal $(2 L) ! / L$ !. Hence, in case $L=M$ there is an infinite class of exceptions.

The following two theorems cover all pairs $(L, M)$ with $L \neq M$ dealt with in the literature up to now.

TheOREM 1. Let $d_{1}, d_{2}, L, M, x$ and $y$ be as in the first paragraph of this section. If

$$
L \in\{2,4\} \quad \text { and } M \text { is odd }
$$

then $\max (x, y) \leq C_{1}$ where $C_{1}$ is some effectively computable number depending only on $d_{1}, d_{2}$ and $M$.

Theorem 2. Let $d_{1}, d_{2}, L, M, x$ and $y$ be as in the first paragraph of this section. Suppose that $\operatorname{gcd}(L, M)>1$ and $L \neq M$. Then

$$
\max (L, M, x, y) \leq C_{2}
$$

where $C_{2}$ is some effectively computable number depending only on $d_{1}, d_{2}$ and $L / M$, unless

$$
\left(d_{1}, d_{2}, L, M, x, y\right) \text { or }\left(d_{2}, d_{1}, M, L, y, x\right) \quad \text { equals } \quad\left(d, 2 d^{2}, 4,2, z, z^{2}+3 d z\right)
$$

for some positive integers $d$ and $z$.

2. The proof of Theorem 1. We shall use the following lemma.

LEMMA 1. Let $P(X)$ be an odd monic polynomial with real coefficients of degree $M>1$ such that for some positive number $v$ there are $(M-1) / 2$ distinct real numbers $\beta$ with $P(\beta)=v, P^{\prime}(\beta)=0$. Then

$$
P(X)=a_{1} T_{M}\left(a_{2} X\right)
$$


where $T_{M}(X)$ is the $M$-th Chebyshev polynomial $\cos (M \arccos X)$ and $a_{1}$ and $a_{2}$ are non-zero real constants.

Proof. As $P$ is odd, there are also $(M-1) / 2$ distinct real numbers $\beta$ with $P(\beta)=-v, P^{\prime}(\beta)=0$. Since $P^{\prime}$ has degree $M-1$, the union of the numbers $\beta$ is the set of roots of $P^{\prime}$ and all the roots of $P^{\prime}$ are simple. It follows that every root of $P^{\prime}$ is a point where $P$ has a local extremum. Since $P$ is monic and odd, the extremum attained for the lowest value of $\beta$ is a maximum $v$, then follows a minimum $-v$, a maximum $v$ and so forth, alternating and ending with a minimum $-v$. Observe that there is a unique point $\beta_{0}$, greater than the largest value where $P$ attains a maximum, such that $P\left(\beta_{0}\right)=v, P^{\prime}\left(\beta_{0}\right)>0$. Of course, $P\left(-\beta_{0}\right)=-v, P^{\prime}\left(-\beta_{0}\right)>0$. Define $\widetilde{P}_{M}(X)=\beta_{0}^{-M} P\left(\beta_{0} X\right)$. Then $\widetilde{P}_{M}(X)$ is a monic polynomial of degree $M$ with $\left|\widetilde{P}_{M}(X)\right| \leq \beta_{0}^{-M} v$ for $|X| \leq 1$ and it assumes the values $\beta_{0}^{-M} v$ and $-\beta_{0}^{-M} v$ each $(M+1) / 2$ times in the interval $[-1,1]$ in alternating way.

Put $\widetilde{T}_{M}(X)=2^{-M+1} T_{M}(X)$. Then $\widetilde{T}_{M}$ has the smallest maximum absolute value on $[-1,1]$ among all monic polynomials of degree $M$ and every monic polynomial $\neq \pm \widetilde{T}_{M}$ has a higher maximum absolute value. (See e.g. [7], pp. 56-57.) Suppose $\widetilde{P}_{M} \neq \widetilde{T}_{M}$. Then $\beta_{0}^{-M} v>\max _{-1 \leq X \leq 1}\left|\widetilde{T}_{M}(X)\right|$, so that $\widetilde{P}_{M}(X)-\widetilde{T}_{M}(X)$ is positive at each point $\beta$ with $\widetilde{P}_{M}(\beta)=\beta_{0}^{-M} v$ and negative at each point $\beta$ with $\widetilde{P}_{M}(\beta)=-\beta_{0}^{-M} v$. Since both $\widetilde{P}_{M}$ and $\widetilde{T}_{M}$ are monic, it follows that $\widetilde{P}_{M}-\widetilde{T}_{M}$ is a polynomial of degree at most $M-1$ which has $M$ sign changes in the interval $[-1,1]$, which is a contradiction. Thus $\widetilde{P}_{M}=\widetilde{T}_{M}$, which implies that

$$
P(X)=2\left(\beta_{0} / 2\right)^{M} T_{M}\left(X / \beta_{0}\right) .
$$

The second lemma is due to Brindza. It is proved by the method of estimating linear forms of logarithms.

Lemma 2. Let $f(X) \in \mathbb{Z}[X], f(X)=a_{0}\left(X-\alpha_{1}\right)^{r_{1}} \ldots\left(X-\alpha_{n}\right)^{r_{n}}$, be a polynomial with distinct roots $\alpha_{1}, \ldots, \alpha_{n}$. Then there exists an effectively computable number $C_{3}$ depending only on $f$ such that the equation $z^{2}=$ $f(y)$ in rational integers $y, z$ implies $\max (|y|,|z|) \leq C_{3}$ unless at most two exponents $r_{j}$ are odd.

Proof. See [2] or [15], Theorem 8.3.

Proof of Theorem 1. Consider equation (1) with $L=2$. Since $x\left(x+d_{1}\right)=\left(x+\frac{1}{2} d_{1}\right)^{2}-\frac{1}{4} d_{1}^{2}$, this implies

$$
z^{2}=4 y\left(y+d_{2}\right) \ldots\left(y+(M-1) d_{2}\right)+d_{1}^{2}
$$

where $z=2 x+d_{1}$. Now consider equation (1) with $L=4$. Then

$$
\left(x^{2}+3 d_{1} x+d_{1}^{2}\right)^{2}-d_{1}^{4}=y\left(y+d_{2}\right) \ldots\left(y+(M-1) d_{2}\right)
$$


whence

$$
z^{2}=y\left(y+d_{2}\right) \ldots\left(y+(M-1) d_{2}\right)+d_{1}^{4}
$$

where $z=x^{2}+3 d_{1} x+d_{1}^{2}$. We conclude that all cases of Theorem 1 can be reduced to an equation

$$
z^{2}=\delta y\left(y+d_{2}\right) \ldots\left(y+(M-1) d_{2}\right)+c^{2}
$$

where $c$ is some positive rational integer and $\delta=1$ or 4 .

We deal first with the cases with $M=3$. In these cases equation (1) is reduced to the elliptic equation

$$
z^{2}=\delta y\left(y+d_{2}\right)\left(y+2 d_{2}\right)+c^{2} .
$$

Put $f(Y)=\delta Y\left(Y+d_{2}\right)\left(Y+2 d_{2}\right)+c^{2}$. According to Lemma 2 we have $\max (|y|,|z|) \leq C_{3}$ where $C_{3}$ depends only on $d_{2}$ and $c$ unless $f$ has a double root $\alpha$. In the latter case we have

$$
0=\delta^{-1} f^{\prime}(\alpha)=3 \alpha^{2}+6 d_{2} \alpha+2 d_{2}^{2},
$$

which implies $\alpha=-d_{2} \pm \frac{d_{2}}{3} \sqrt{3}$. Hence

$$
0=f(\alpha)=\delta\left(\alpha^{3}+3 d_{2} \alpha^{2}+2 d_{2}^{2} \alpha\right)+c^{2}=\mp \frac{2}{9} \delta d_{2}^{3} \sqrt{3}+c^{2} .
$$

Since $\delta, c$ and $d_{2}$ are rational, this implies $d_{2}=0$, which is a contradiction. So we obtain $\max (x, y) \leq C_{4}$ where $C_{4}$ is some computable number depending only on $d_{1}$ and $d_{2}$.

We are left with the cases $L \in\{2,4\}$ and $M$ is odd, $M \geq 5$. Here we consider the hyperelliptic equation $z^{2}=f(y)$ where

$$
f(Y):=\delta Y\left(Y+d_{2}\right) \ldots\left(Y+(M-1) d_{2}\right)+c^{2}
$$

and $c$ is some positive rational integer. According to Lemma 2 we have $\max (y, z) \leq C_{3}$ where $C_{3}$ depends only on $c, d_{2}$ and $M$, unless $f$ has exactly one root of odd order. In the latter case we may assume without loss of generality that

$$
f(Y)=\delta\left(Y-\alpha_{1}\right)^{r_{1}}\left(Y-\alpha_{2}\right)^{r_{2}} \ldots\left(Y-\alpha_{n}\right)^{r_{n}}
$$

with $\alpha_{1}, \ldots, \alpha_{n}$ distinct roots, $r_{1}$ odd and $r_{2}, \ldots, r_{n}$ even. Since $f(Y)-c^{2}$ has $M$ distinct real roots, the roots of $f^{\prime}$ are real and simple by Rolle's theorem. Thus $r_{1}=1, r_{2}=\ldots=r_{n}=2$. Therefore $M=2 n-1$ and $f$ has $(M-1) / 2$ double roots $\alpha_{2}, \ldots, \alpha_{n}$.

Consider the polynomial

$$
g(Y):=\left(Y-\frac{M-1}{2}\right)\left(Y-\frac{M-3}{2}\right) \ldots\left(Y+\frac{M-3}{2}\right)\left(Y+\frac{M-1}{2}\right) .
$$

Observe that $g$ is an odd function and that

$$
f(Y)=\delta d_{2}^{M} g\left(\frac{Y}{d_{2}}+\frac{M-1}{2}\right)+c^{2} .
$$


Since $f$ has $(M-1) / 2$ double roots, the polynomial $g$ has $(M-1) / 2$ distinct real values $\beta$ with $g(\beta)=-\delta c^{2} d_{2}^{-M}, g^{\prime}(\beta)=0$. Since $g$ is odd, Lemma 1 gives that the function $g$ is of the form $g(Y)=a_{1} T_{M}\left(a_{2} Y\right)$ where $T_{M}$ is the $M$ th Chebyshev polynomial and $a_{1}$ and $a_{2}$ are non-zero real constants. This implies that

$$
f(Y)=\delta a_{1} d_{2}^{M} T_{M}\left(\frac{a_{2}}{d_{2}} Y+\frac{a_{2}(M-1)}{2}\right)+c^{2} .
$$

We infer from (7) that

$$
Y\left(Y+d_{2}\right) \ldots\left(Y+(M-1) d_{2}\right)=a_{1} d_{2}^{M} T_{M}\left(\frac{a_{2}}{d_{2}} Y+\frac{a_{2}(M-1)}{2}\right) .
$$

However, it follows from the definition of Chebyshev polynomials that for $M>3$ the roots of $T_{M}$ are not equidistant, which yields a contradiction. We conclude that $\max (x, y) \leq C_{5}$ where $C_{5}$ is some number depending only on $d_{1}, d_{2}$ and $M$.

3. Lemmas for the proof of Theorem 2. Using the notation of the first paragraph of the introduction we rewrite (1) as follows:

$$
x\left(x+d_{1}\right) \ldots\left(x+(l k-1) d_{1}\right)=y\left(y+d_{2}\right) \ldots\left(y+(m k-1) d_{2}\right) .
$$

Without loss of generality we shall assume that $l<m$. We shall frequently use the Vinogradov symbol $\ll$ and then tacitly assume that the implied constants are effectively computable positive numbers depending only on $d_{1}, d_{2}, l$ and $m$. Note that $l$ and $m$ are completely determined by $L / M$.

LEMma 3. $k \ll \log (x+1)$.

Proof. We assume that $k$ is larger than some suitable number depending only on $d_{1}, d_{2}, l$ and $m$. We express this by saying that $k$ is taken sufficiently large. Let $p$ be the smallest prime number which does not divide $d_{1} d_{2}$. Then

$$
\operatorname{ord}_{p}\left(y\left(y+d_{2}\right) \ldots\left(y+(m k-1) d_{2}\right)\right) \geq\left[\frac{m k}{p}\right]+\left[\frac{m k}{p^{2}}\right]+\ldots
$$

On the other hand,

$$
\begin{aligned}
\operatorname{ord}_{p}\left(x\left(x+d_{1}\right) \ldots(x\right. & \left.\left.+(l k-1) d_{1}\right)\right) \\
& \leq \max _{i=0, \ldots, l k-1} \operatorname{ord}_{p}\left(x+i d_{1}\right)+\left[\frac{l k}{p}\right]+\left[\frac{l k}{p^{2}}\right]+\ldots
\end{aligned}
$$

It now follows from (8) and $l<m$ that, for $k$ sufficiently large,

$$
k \ll\left[\frac{m k}{p}\right]-\left[\frac{l k}{p}\right] \leq \max _{i=0, \ldots, l k-1} \operatorname{ord}_{p}\left(x+i d_{1}\right) \ll \log \left(x+k d_{1}\right) .
$$

Hence $k \ll \log (x+1)$.

Lemma 4. $x^{l}-y^{m} \ll y^{m-1} \log (y+1), y^{m}-x^{l} \ll x^{l-1} \log (x+1)$. 
Proof. We have, by (8), $y^{k m} \leq\left(x+k l d_{1}\right)^{k l}$. Hence, by Lemma 3 , $y^{m / l}-x \leq k l d_{1} \ll \log (x+1)$. This implies $y^{m}-x^{l} \ll x^{l-1} \log (x+1)$. For the proof of the first inequality we derive from (8) and Lemma 3 as above that $x^{l / m}-y \ll \log (x+1)$, which implies that $\log (x+1) \ll \log (y+1)$ and the first inequality follows.

The following inequalities follow immediately from Lemmas 3 and 4:

$$
\begin{gathered}
k \ll \log (y+1), \\
x^{l} \ll y^{m}, \quad y^{m} \ll x^{l}, \quad\left|x^{l}-y^{m}\right| \ll \min \left(x^{l}, y^{m}\right) \frac{\log y}{y} .
\end{gathered}
$$

Because of Lemma 3, (9) and (10) we may assume that $x$ and $y$ are larger than some suitable number depending only on $d_{1}, d_{2}, l$ and $m$. We express this by saying that $x$ (or $y$ ) is taken sufficiently large. From now onwards, we shall assume without reference that $x$ and $y$ are sufficiently large.

We adopt the notation of [10] in slightly modified form. We define positive integers $A_{j}(\nu, k)$ for $\nu \in\{l, m\}$ by

$$
z(z+1) \ldots(z+\nu k-1)=\sum_{j=0}^{\nu k-1} A_{j}(\nu, k) z^{\nu k-j} .
$$

Further we determine rational numbers $B_{j}(\nu, k)$ and $H_{j}(\nu, k)$ such that

$$
\left(z^{\nu}+B_{1}(\nu, k) z^{\nu-1}+\ldots+B_{\nu}(\nu, k)\right)^{k}=\sum_{j=0}^{\nu k} H_{j}(\nu, k) z^{\nu k-j}
$$

satisfies

$$
H_{j}(\nu, k)=A_{j}(\nu, k) \quad \text { for } 0 \leq j \leq \nu .
$$

We introduce the notation

$$
G_{j}(\nu, k)=A_{j}(\nu, k)-H_{j}(\nu, k) \quad \text { for } 0<j \leq \nu k .
$$

Lemma 5. There exist effectively computable absolute constants $c_{1}$ and $c_{2}$ such that

$$
\begin{array}{ll}
A_{j}(\nu, k) \leq(\nu k)^{2 j} & \text { for } 0 \leq j<\nu k, \\
B_{j}(\nu, k) \leq c_{1}^{j / 2}(\nu k)^{2 j} & \text { for } 1 \leq j \leq \nu, \\
\left|G_{j}(\nu, k)\right| \leq c_{2}^{j \sqrt{\nu}}(\nu k)^{2 j} & \text { for } 0<j \leq \nu k, \\
k^{2 j-1} B_{j}(\nu, k) \in \mathbb{Z} & \text { for } 1 \leq j \leq \nu, \\
k^{2 j-1} G_{j}(\nu, k) \in \mathbb{Z} & \text { for } 0<j \leq \nu k .
\end{array}
$$


Proof. (a) $A_{j}(\nu, k) \leq\left(\begin{array}{c}\nu k-1 \\ j\end{array}\right)(\nu k)^{j}<(\nu k)^{2 j}$.

(b) As the proof of [10], Lemma 1.

(c) As the proof of [10], Lemma 2.

(d), (e) As the proof of [10], Lemma 3.

Put

$$
\begin{array}{cl}
L_{j}=B_{j}(l, k) d_{1}^{j} & \text { for } 1 \leq j \leq l, \\
M_{j}=B_{j}(m, k) d_{2}^{j} & \text { for } 1 \leq j \leq m, \\
L_{j}^{*}=G_{j}(l, k) d_{1}^{j} & \text { for } 1 \leq j \leq l k, \\
M_{j}^{*}=G_{j}(m, k) d_{2}^{j} & \text { for } 1 \leq j \leq m k .
\end{array}
$$

Then

$$
\begin{aligned}
& x\left(x+d_{1}\right) \ldots\left(x+(l k-1) d_{1}\right) \\
& \quad=\left(x^{l}+L_{1} x^{l-1}+\ldots+L_{l}\right)^{k}+L_{l+1}^{*} x^{k l-l-1}+L_{l+2}^{*} x^{k l-l-2}+\ldots
\end{aligned}
$$

and

$$
\begin{aligned}
& y\left(y+d_{2}\right) \ldots\left(y+(m k-1) d_{2}\right) \\
& =\left(y^{m}+M_{1} y^{m-1}+\ldots+M_{m}\right)^{k} \\
& +M_{m+1}^{*} y^{k m-m-1}+M_{m+2}^{*} y^{k m-m-2}+\ldots .
\end{aligned}
$$

\section{LEMMA 6.}

$$
x^{l}+L_{1} x^{l-1}+\ldots+L_{l}=y^{m}+M_{1} y^{m-1}+\ldots+M_{m} .
$$

Proof. By (13), (14), (8), (12) and Lemma 5(c),

$$
\begin{aligned}
D: & =\left|\left(x^{l}+L_{1} x^{l-1}+\ldots+L_{l}\right)^{k}-\left(y^{m}+M_{1} y^{m-1}+\ldots+M_{m}\right)^{k}\right| \\
& =\left|\sum_{i=l+1}^{l k} L_{i}^{*} x^{l k-i}-\sum_{j=m+1}^{m k} M_{j}^{*} y^{m k-j}\right| \\
& \leq \sum_{i=l+1}^{l k} c_{2}^{i \sqrt{l}}\left(l k d_{1}\right)^{2 i} x^{l k-i}+\sum_{j=m+1}^{m k} c_{2}^{j \sqrt{m}}\left(m k d_{2}\right)^{2 j} y^{m k-j} .
\end{aligned}
$$

By Lemma 3 and (9), we obtain

$$
\begin{aligned}
D & \leq \frac{\left(l k d_{1}\right)^{2 l+2} c_{2}^{(l+1) \sqrt{l}} x^{l k-l-1}}{1-c_{2}^{\sqrt{l}}\left(l k d_{1}\right)^{2} / x}+\frac{\left(m k d_{2}\right)^{2 m+2} c_{2}^{(m+1) \sqrt{m}} y^{m k-m-1}}{1-c_{2}^{\sqrt{m}}\left(m k d_{2}\right)^{2} / y} \\
& \ll\left(\frac{k^{2}}{x}\right)^{l+1} x^{l k}+\left(\frac{k^{2}}{y}\right)^{m+1} y^{m k} .
\end{aligned}
$$


On the other hand, we have $L_{1}>0, M_{1}>0$ and

$$
\begin{aligned}
D= & \left|\left(x^{l}+L_{1} x^{l-1}+\ldots+L_{l}\right)^{k}-\left(y^{m}+M_{1} y^{m-1}+\ldots+M_{m}\right)^{k}\right| \\
\geq & \left|\left(x^{l}+L_{1} x^{l-1}+\ldots+L_{l}\right)-\left(y^{m}+M_{1} y^{m-1}+\ldots+M_{m}\right)\right| \\
& \times \min \left(x^{l(k-1)}, y^{m(k-1)}\right) .
\end{aligned}
$$

Suppose (15) does not hold. Then, by (11), Lemma 5(d) and $l<m$, it follows that $D \geq w^{k-1} / k^{2 m-1}$ with $w=\min \left(x^{l}, y^{m}\right)$. On combining the lower and upper bound for $D$ we obtain, using the fact that $l<m$ and $x \gg y$ by Lemma 4,

$$
w^{k-1} \leq k^{2 m-1} D \ll \frac{k^{4 m+1}}{y} \max \left(x^{l k-l}, y^{m k-m}\right) .
$$

Hence, by (9) and (10),

$$
w^{k-1} \ll \frac{k^{4 m+1}}{y}\left(w+\left|x^{l}-y^{m}\right|\right)^{k-1} \ll \frac{(\log y)^{4 m+1}}{y} w^{k-1}\left(1+\frac{\log y}{y}\right)^{k-1} .
$$

This implies, by (9),

$$
\log y \ll \log \log y+k \log \left(1+\frac{\log y}{y}\right) \ll \log \log y+\frac{(\log y)^{2}}{y} .
$$

This proves that $y \ll 1$, which is a contradiction.

LEMma 7. We have

$$
L_{i}^{*}=0 \quad \text { for } l<i<2 l
$$

and

$$
M_{j}^{*}=0 \quad \text { for } m<j<2 m .
$$

Proof. Define $I$ and $J$ by

$$
L_{l+1}^{*}=\ldots=L_{I-1}^{*}=0, \quad L_{I}^{*} \neq 0, \quad M_{m+1}^{*}=\ldots=M_{J-1}^{*}=0, \quad M_{J}^{*} \neq 0 .
$$

By (15), (8), (13) and (14), we obtain $\sum_{i=I}^{l k} L_{i}^{*} x^{l k-i}=\sum_{j=J}^{m k} M_{j}^{*} y^{m k-j}$. Therefore, by (10), it suffices to show that either $A_{l+1}=\ldots=A_{2 l-1}=0$ or $B_{m+1}=\ldots=B_{2 m-1}=0$. Suppose that this assertion is false. Then we can take $l<I<2 l$ and $m<J<2 m$. Observe that $m I=l J$ and $\operatorname{gcd}(l, m)=1$ imply $l \mid I$, a contradiction. We prove the lemma when $m I<l J$ and the proof for the case $m I>l J$ is similar. We have

$$
m I \leq l J-1 .
$$


Hence, by (12), Lemma 5(c), Lemma 3 and (9),

$$
\begin{aligned}
\left|L_{I}^{*} x^{l k-I}\right| & \leq \sum_{i=I+1}^{l k}\left|L_{i}^{*}\right| x^{l k-i}+\sum_{j=J}^{m k}\left|M_{j}^{*}\right| y^{m k-j} \\
& \leq \sum_{i=I+1}^{l k} c_{2}^{i \sqrt{l}}\left(l k d_{1}\right)^{2 i} x^{l k-i}+\sum_{j=J}^{m k} c_{2}^{j \sqrt{m}}\left(m k d_{2}\right)^{2 j} y^{m k-j} \\
& \leq 2 c_{2}^{(I+1) \sqrt{l}} \frac{\left(l k d_{1}\right)^{2 I+2}}{x^{I+1}} x^{l k}+2 c_{2}^{J \sqrt{m}} \frac{\left(m k d_{2}\right)^{2 J}}{y^{J}} y^{m k} .
\end{aligned}
$$

By (12), Lemma $5(\mathrm{e})$ and $L_{I}^{*} \neq 0$, we have $\left|L_{I}^{*}\right| \geq k^{-2 I}$. Hence, by $I<2 l$, Lemma 3 and (10),

$$
\begin{aligned}
1 & \leq 2 c_{2}^{(I+1) \sqrt{l}} k^{2 I} \frac{\left(l k d_{1}\right)^{2 I+2}}{x}+2 c_{2}^{J \sqrt{m}} k^{2 I} \frac{\left(m k d_{2}\right)^{2 J}}{y^{J-m I / l}}\left(\frac{y^{m}}{x^{l}}\right)^{k-I / l} \\
& \leq 2 \frac{\left(c_{2}^{\sqrt{l}} l k d_{1}\right)^{8 l}}{x}+2 \frac{c_{2}^{J \sqrt{m}} k^{4 l}\left(m k d_{2}\right)^{2 J}}{y^{J-m I / l}}\left(1+\frac{\left|y^{m}-x^{l}\right|}{x^{l}}\right)^{k} \\
& \leq \frac{1}{2}+2 \frac{c_{2}^{J \sqrt{m}} k^{4 l}\left(m k d_{2}\right)^{2 J}}{y^{J-m I / l}}\left(1+\frac{c_{3} \log y}{y}\right)^{k}
\end{aligned}
$$

for some number $c_{3}$ depending only on $d_{1}, d_{2}, l$ and $m$. Since $J<2 m$, (18) implies, by (17),

$$
y^{1 / l} \leq c_{2}^{J \sqrt{m}}\left(m k d_{2}\right)^{10 m}\left(1+\frac{c_{3} \log y}{y}\right)^{k} .
$$

By (19) and (9)

$$
\log y \ll \log k+k \log \left(1+\frac{c_{3} \log y}{y}\right) \ll \log \log y+\frac{(\log y)^{2}}{y} .
$$

It is clear from $(20)$ that $y \ll 1$, a contradiction.

The next lemma is due to R. Balasubramanian.

Lemma 8. Let $m>2$. There exists an effectively computable number $C_{6}$ depending only on $m$ and an integer $q$ with $m<q<2 m$ such that

$$
G_{q}(m, k) \neq 0 \quad \text { for } k \geq C_{6} .
$$

Pro of. See [10], Lemma 7.

Lemma 9. Suppose that $f(X)$ and $g(Y)$ are polynomials of positive degree with rational numbers as coefficients. Assume that the degrees of $f$ and $g$ are relatively prime. Then $f(X)-g(Y)$ is irreducible over the rationals.

Proof. This is due to Ehrenfeucht [4]. Cf. [14], p. 94 and [3]. 
4. Proof of Theorem 2. It suffices to show that equation (8) with $k>1$ and $l<m$ implies that $\max (k, x, y) \leq C_{2}$. By the result of Saradha and Shorey [10] mentioned in the introduction we may assume $m>2$. By Lemma 3 and (10) we may take $x$ and $y$ sufficiently large so that (16) holds. Then, by (12), Lemma 8 and $m>2$, we have $k \ll 1$. We may therefore assume that $k$ is fixed. Hence $L_{1}, \ldots, L_{l}$ and $M_{1}, \ldots, M_{m}$ are fixed. If

$$
X\left(X+d_{1}\right) \ldots\left(X+(l k-1) d_{1}\right)-Y\left(Y+d_{2}\right) \ldots\left(Y+(m k-1) d_{2}\right)
$$

and

$$
\left(X^{l}+L_{1} X^{l-1}+\ldots+L_{l}\right)-\left(Y^{m}+M_{1} Y^{m-1}+\ldots+M_{m}\right)
$$

have no non-constant common factor, then the resultant of both polynomials with respect to $X$ is a non-zero polynomial in $Y$ which is a linear combination of the polynomials (22) and (23). Since every sufficiently large solution $(x, y)$ of (8) is also a solution of (15), it follows that $y$ is a zero of the resultant. This implies that $y$ is bounded. This contradicts our assumption that $y$ is sufficiently large. If (22) and (23) have a non-constant common factor, then we see from Lemma 9 that (22) has to be divisible by (23).

Put $g(Y)=Y^{m}+M_{1} Y^{m-1}+\ldots+M_{m}$. By taking $Y=0,-d_{2},-2 d_{2}, \ldots$, $-(m k-1) d_{2}$ we find that each polynomial

$$
f_{j}(X):=X^{l}+L_{1} X^{l-1}+\ldots+L_{l}-g\left(-j d_{2}\right) \quad(j=0,1, \ldots, m k-1)
$$

is a divisor of

$$
X\left(X+d_{1}\right) \ldots\left(X+(l k-1) d_{1}\right) .
$$

However, $g$ can assume each value at most $m$ times. So in $0,-d_{2}, \ldots$, $-(m k-1) d_{2}$ the polynomial $g$ attains at least $k$ distinct values. To each value corresponds a polynomial $f_{j}(X)$ of degree $l$. Since $X\left(X+d_{1}\right) \ldots$ $\ldots\left(X+(l k-1) d_{1}\right)$ is a polynomial of degree $l k$ and any two distinct polynomials $f_{j}$ are coprime (their difference is constant), there are at most $k$ distinct polynomials $f_{j}$. Thus the polynomials $\left\{f_{j}\right\}_{j=0}^{m k-1}$ split into $k$ classes of size $m$ such that within a class the polynomials are identical and any two polynomials from different classes are distinct.

First we consider the case where $m$ is odd. We have shown that at the points $\left\{-j d_{2} \mid 0 \leq j<m k\right\}$ the monic polynomial $g$ of odd degree $m>2$ attains exactly $k$ distinct values and each value precisely $m$ times. Denote these values by $v_{1}<v_{2}<\ldots<v_{k}$ and the points where $g$ attains the value $v_{i}$ by $-j_{i, 1} d_{2}<-j_{i, 2} d_{2}<\ldots<-j_{i, m} d_{2}(i=1, \ldots, k)$. By Rolle's theorem each interval $\left(-j_{i, h} d_{2},-j_{i, h+1} d_{2}\right)$ contains a zero of $g^{\prime}$. Since $g^{\prime}$ has only $m-1$ zeros, $z_{1}, z_{2}, \ldots, z_{m-1}$ say, these zeros are distinct and simple and

$$
\begin{array}{r}
-j_{i, 1} d_{2}<z_{1}<-j_{i, 2} d_{2}<z_{2}<\ldots<-j_{i, m-1} d_{2}<z_{m-1}<-j_{i, m} d_{2} \\
(i=1, \ldots, k) .
\end{array}
$$


The polynomial $g$ is increasing on $\left(-\infty, z_{1}\right)$, decreasing on $\left(z_{1}, z_{2}\right)$, increasing on $\left(z_{2}, z_{3}\right), \ldots$, increasing on $\left(z_{m-1}, \infty\right)$. It follows that the set $\left\{-j_{i, 1} d_{2} \mid 1 \leq\right.$ $i \leq k\}$ consists of the $k$ extreme negative points $\left\{-j d_{2} \mid(m-1) k \leq j \leq\right.$ $m k-1\}$ and, more precisely, $j_{1,1}=m k-1, j_{2,1}=m k-2, \ldots, j_{k, 1}=m k-k$. Further, we have the following scheme in case $m$ is odd ( $\downarrow$ indicates $g$ is increasing, $\uparrow$ indicates $g$ is decreasing):

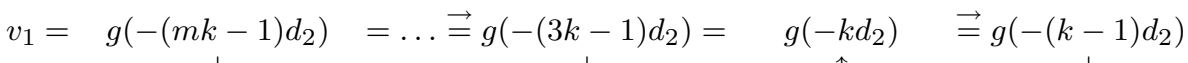

$$
\begin{aligned}
& v_{2}=g\left(-(m k-2) d_{2}\right) \quad=\ldots=g\left(-(3 k-2) d_{2}\right)=g\left(-(k+1) d_{2}\right)=g\left(-\left(\begin{array}{c}
\left.\downarrow-2) d_{2}\right) \\
\downarrow
\end{array}\right)\right. \\
& \begin{array}{cccc}
\vdots & \vdots & \vdots & \vdots \\
\downarrow & \downarrow & \uparrow & \vdots \\
v_{k-1}=g\left(-(m k-k+1) d_{2}\right) & =\ldots=g\left(-(2 k+1) d_{2}\right) & =g\left(-(2 k-2) d_{2}\right)= & g\left(-d_{2}\right) \\
\downarrow & \downarrow & \uparrow & \downarrow
\end{array} \\
& v_{k}=g\left(-(m k-k) d_{2}\right) \quad \overrightarrow{=} \ldots=g\left(-2 k d_{2}\right) \quad \overrightarrow{=} g\left(-(2 k-1) d_{2}\right)=\quad g(0)
\end{aligned}
$$

Hence we have

$$
\begin{array}{r}
g(Y)=Y\left(Y+(2 k-1) d_{2}\right)\left(Y+2 k d_{2}\right)\left(Y+(4 k-1) d_{2}\right) \ldots \\
\ldots\left(Y+(m k-k) d_{2}\right)+v_{k} \\
=\left(Y+d_{2}\right)\left(Y+(2 k-2) d_{2}\right)\left(Y+(2 k+1) d_{2}\right)\left(Y+(4 k-2) d_{2}\right) \ldots \\
\ldots\left(Y+(m k-k+1) d_{2}\right)+v_{k-1} .
\end{array}
$$

By putting $Y=0$ and $Y=-(2 k-1) d_{2}$ in the above equality we have

$$
\begin{aligned}
v_{k}-v_{k-1} & =d_{2}^{m} \cdot 1 \cdot(2 k-2)(2 k+1)(4 k-2) \ldots(m k-k+1) \\
& =d_{2}^{m} \cdot(-2 k+2)(-1)(2)(2 k-1) \ldots(m k-3 k+2),
\end{aligned}
$$

which is impossible since $m \geq 3$.

If $m$ is even, a similar reasoning yields the following scheme:

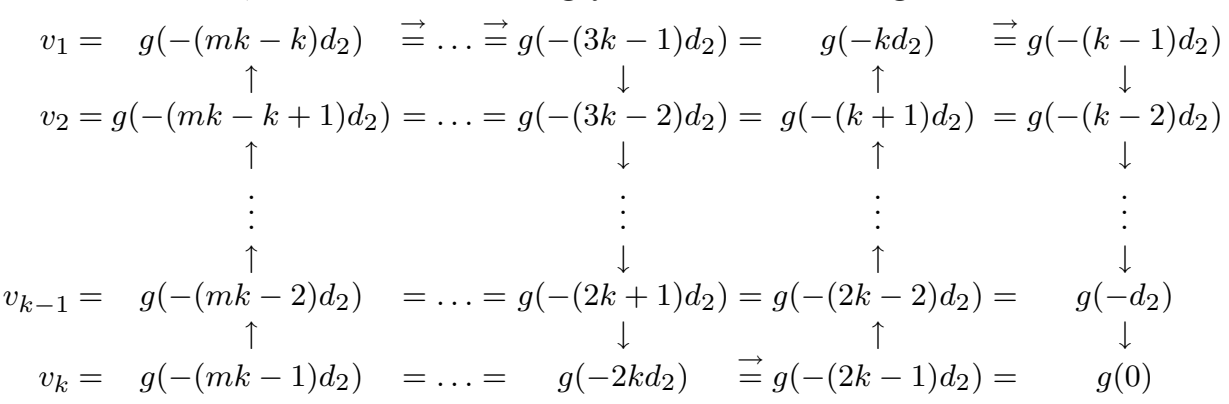

Hence we have

$$
\begin{array}{r}
g(Y)=Y\left(Y+(2 k-1) d_{2}\right)\left(Y+2 k d_{2}\right)\left(Y+(4 k-1) d_{2}\right) \ldots \\
\ldots\left(Y+(m k-1) d_{2}\right)+v_{k} \\
=\left(Y+d_{2}\right)\left(Y+(2 k-2) d_{2}\right)\left(Y+(2 k+1) d_{2}\right)\left(Y+(4 k-2) d_{2}\right) \ldots \\
\ldots\left(Y+(m k-2) d_{2}\right)+v_{k-1}
\end{array}
$$


By putting $Y=0$ and $Y=-(2 k-1) d_{2}$ in the above equality we have

$$
\begin{aligned}
v_{k}-v_{k-1} & =d_{2}^{m} \cdot 1 \cdot(2 k-2)(2 k+1)(4 k-2) \ldots(m k-2) \\
& =d_{2}^{m} \cdot(-2 k+2)(-1)(2)(2 k-1) \ldots(m k-2 k-1),
\end{aligned}
$$

which is impossible since $m \geq 4$. In fact, the above argument is also valid when $m=2$ and $k \geq 3$.

\section{References}

[1] D. W. Boyd and H. H. Kisilevsky, The diophantine equation $u(u+1)(u+2)(u+$ $3)=v(v+1)(v+2)$, Pacific J. Math. 40 (1972), 23-32.

[2] B. Brindza, On S-integral solutions of the equation $y^{m}=f(x)$, Acta Math. Hungar. 44 (1984), 133-139.

[3] J. W. S. Cassels, Factorization of polynomials in several variables, in: Proc. 15th Scandinavian Congress, Oslo 1968, Lecture Notes in Math. 118, Springer, 1970, $1-17$.

[4] A. Ehrenfeucht, A criterion for absolute irreducibility of polynomials, Prace Mat. 2 (1958), 167-169 (in Polish).

[5] R. A. MacLeod and I. Barrodale, On equal products of consecutive integers, Canad. Math. Bull. 13 (1970), 255-259.

[6] L. J. Mordell, On the integer solutions of $y(y+1)=x(x+1)(x+2)$, Pacific J. Math. 13 (1963), 1347-1351.

[7] T. J. Rivlin, The Chebyshev Polynomials, Wiley, New York, 1974.

[8] N. Saradha and T. N. Shorey, On the ratio of two blocks of consecutive integers, Proc. Indian Acad. Sci. (Math. Sci.) 100 (1990), 107-132.

[9] - - - On the equation $(x+1) \ldots(x+k)=(y+1) \ldots(y+m k)$ with $m=3,4$, Indag. Math. 2 (1991), 489-510.

[10] - - - On the equation $(x+1) \ldots(x+k)=(y+1) \ldots(y+m k)$, ibid. 3 (1992), 79-90.

[11] - - - On the equation $x(x+d) \ldots(x+(k-1) d)=y(y+d) \ldots(y+(m k-1) d)$, ibid. 3 (1992), 237-242.

$[12] \quad-,-$, On the equation $x\left(x+d_{1}\right) \ldots\left(x+(k-1) d_{1}\right)=y\left(y+d_{2}\right) \ldots\left(y+(m k-1) d_{2}\right)$, Proc. Indian Acad. Sci. (Math. Sci.) 104 (1994).

[13] N. Saradha, T. N. Shorey and R. Tijdeman, On arithmetic progressions of equal lengths with equal products, Math. Proc. Cambridge Philos. Soc., to appear.

[14] A. Schinzel, Selected Topics on Polynomials, University of Michigan Press, Ann Arbor, 1982.

[15] T. N. Shorey and R. Tijdeman, Exponential Diophantine Equations, Cambridge University Press, 1986.

SCHOOL OF MATHEMATICS

TATA INSTITUTE OF FUNDAMENTAL RESEARCH HOMI BHABHA ROAD

BOMBAY 400005

INDIA
MATHEMATICAL INSTITUTE LEIDEN UNIVERSITY P.O. BOX 9512 2300 RA LEIDEN THE NETHERLANDS 